\title{
A Higher Sensory Brain Region Is Involved in Reversing Reinforcement-Induced Vocal Changes in a Songbird
}

\author{
Alessandro Canopoli, ${ }^{1,2}$ Joshua A. Herbst, ${ }^{1,2}$ and Richard H.R. Hahnloser ${ }^{1,2}$ \\ ${ }^{1}$ Institute of Neuroinformatics, University of Zurich and ETH Zurich, 8057 Zurich, Switzerland and ${ }^{2}$ Neuroscience Center Zurich (ZNZ), 8057 Zurich, \\ Switzerland
}

Many animals exhibit flexible behaviors that they can adjust to increase reward or avoid harm (learning by positive or aversive reinforcement). But what neural mechanisms allow them to restore their original behavior (motor program) after reinforcement is withdrawn? One possibility is that motor restoration relies on brain areas that have a role in memorization but no role in either motor production or in sensory processing relevant for expressing the behavior and its refinement.

We investigated the role of a higher auditory brain area in the songbird for modifying and restoring the stereotyped adult song. We exposed zebra finches to aversively reinforcing white noise stimuli contingent on the pitch of one of their stereotyped song syllables. In response, birds significantly changed the pitch of that syllable to avoid the aversive reinforcer. After we withdrew reinforcement, birds recovered their original song within a few days. However, we found that large bilateral lesions in the caudal medial nidopallium (NCM, a high auditory area) impaired recovery of the original pitch even several weeks after withdrawal of the reinforcing stimuli. Because NCM lesions spared both successful noise-avoidance behavior and birds' auditory discrimination ability, our results show that NCM is not needed for directed motor changes or for auditory discriminative processing, but is implied in memorizing or recalling the memory of the recent song target.

\section{Introduction}

Many forms of sensorimotor learning involve a trial and error process of motor explorations to approximate a sensory target. In the case of vocalizations, motor learning can occur long after the sensory target has last been experienced (Marler and Tamura, 1964; Funabiki and Konishi, 2003), revealing a long-lasting influence of sensory memories on development and maintenance of motor performance. Vocal learners also rely on the ability to process self-generated auditory input, as shown, for instance, by the disruptive effects of deafening or auditory feedback perturbation on human speech (Lane and Webster, 1991; Svirsky et al., 1992; Houde and Jordan, 1998) and birdsong (Nordeen and Nordeen, 1992; Leonardo and Konishi, 1999; Brainard and Doupe, 2000).

Very few animal models are suited for studying auditory-vocal memory. Higher mammals such as nonhuman primates and dogs not only limit their vocal output to innate vocalizations (Hauser et al., 2002), but they also perform poorly in long-term memory tests when the sensory modality is auditory (Fritz et al.,

\footnotetext{
Received Jan. 21, 2014; revised March 14, 2014; accepted March 26, 2014.

Author contributions: A.C., J.A.H., and R.H.R.H. designed research; A.C. performed research; A.C. analyzed data; A.C., J.A.H., and R.H.R.H. wrote the paper.

This work was funded by the Swiss National Science Foundation (Grant 31003A_127024) and by the European Research Council under the European Community's Seventh Framework Programme (ERC Grant AdG 268911 FP7/ 2007-2013). We thank Anna Stepien, Anja Zai, Michael Griesser, and Alexander Hanuschkin for helpful comments on this manuscript and Franziska Baumann-Klausener and Simone Rickauer for technical assistance.

The authors declare no competing financial interests.

Correspondence should be addressed to Richard H.R. Hahnloser, Institute of Neuroinformatics, University of Zurich and ETH Zurich, 8057 Zurich, Switzerland. E-mail: rich@ini.phys.ethz.ch.

DOI:10.1523/JNEUROSCI.0266-14.2014

Copyright $\odot 2014$ the authors $\quad 0270-6474 / 14 / 347018-09 \$ 15.00 / 0$
}

2005; Scott et al., 2012). In contrast, vocal learners such as songbirds rely heavily on audition and perform well in standard auditory memory tasks, even when compared with humans (Zokoll et al., 2008; Comins and Gentner, 2010). To study the neural mechanisms underlying sensory memory and motor plasticity we perform brain lesions in zebra finches and characterize the lesion effects on their ability to selectively change and restore their songs.

We are interested in the memory role of the caudal medial nidopallium (NCM), a secondary auditory brain area that is often compared with human auditory association cortex (Reiner et al., 2004). Suppression of protein synthesis in NCM and neighboring areas in juvenile birds during song tutoring significantly impairs the quality of subsequent song copying (London and Clayton, 2008). NCM lesions in adults do not affect song production but lead to a reduction of behavioral preference for tutor song (Gobes and Bolhuis, 2007). In combination with correlative evidence (Chew et al., 1995, 1996; Bolhuis et al., 2000; Phan et al., 2006; Moorman et al., 2012), these results suggest that NCM may be necessary for storing or retrieving a memory of a song target. However, it remains unknown whether such target memory plays a role in maintaining adult song.

Here we apply an operant conditioning paradigm in which we deliver a loud white noise stimulus to induce adult zebra finches to change the pitch of one of their song syllables away from its normal range (Tumer and Brainard, 2007; Andalman and Fee, 2009; Warren et al., 2011). When we end white noise playback, birds spontaneously restore baseline pitch levels within a few days, thus displaying an intact memory of their baseline pitch. However, when the end of noise playback is coupled with bilat- 
A
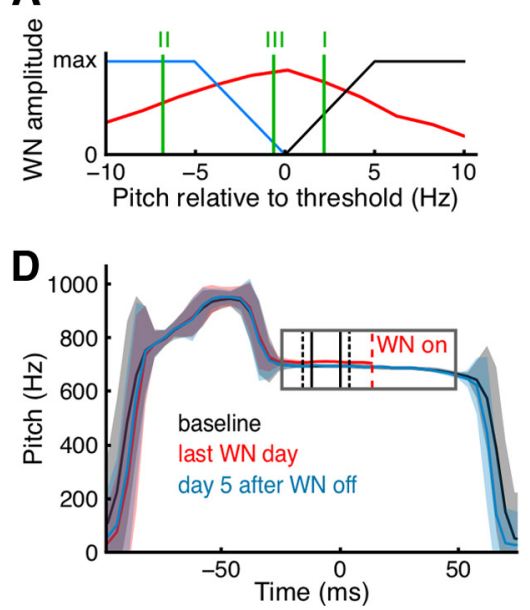
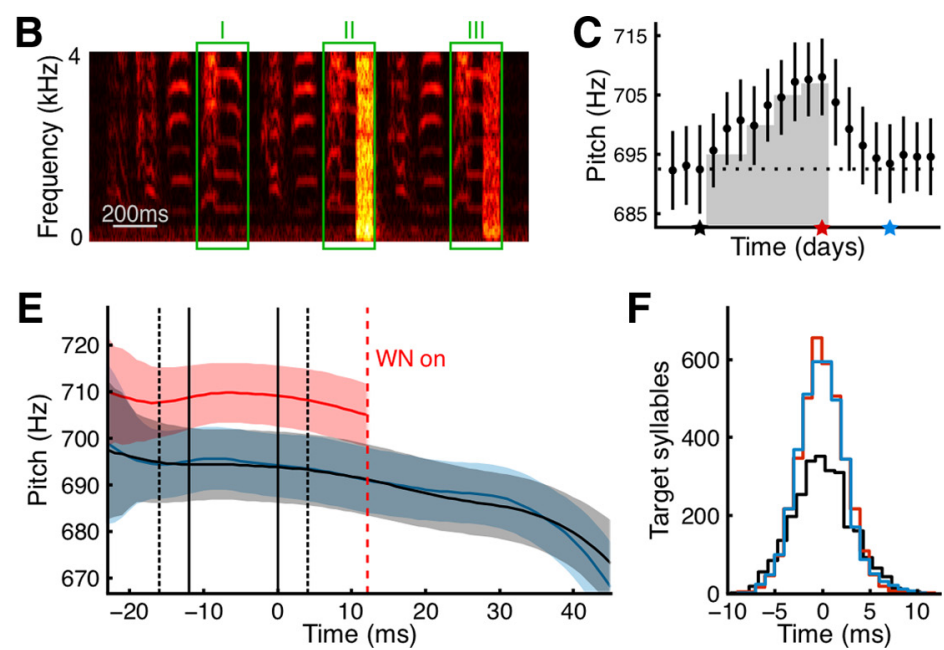

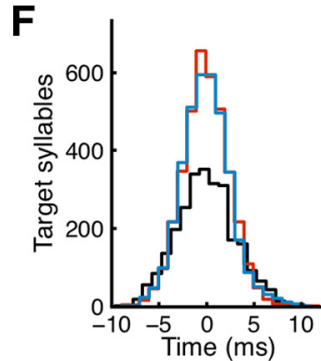

Figure 1. Pitch reinforcement and recovery. $A$, We drove the pitch of zebra finch song syllables up (down) by broadcasting WN stimuli when pitch was below (above) a manually set threshold. Shown is the WN amplitude as a function of pitch on days with aversive reinforcement of low-pitch (blue) and of high-pitch (black) syllables. We indicate the pitches of three syllables by green vertical lines (I, II, and III); these syllables are also delineated by green boxes in $\boldsymbol{B}$. For reference, overlaid is the pitch distribution (red) produced during the last day of pitch reinforcement (this day is highlighted by a red star in ( and indicated by red lines in $\boldsymbol{D}-\boldsymbol{F}$ ). $\boldsymbol{B}$, Sample sound spectrogram of a zebra finch song bout (high sound intensities shown in yellow and low intensities in red-black). The first (I) highlighted target syllable is an escape, the second (II), triggered full amplitude WN; and the third (III), triggered low-amplitude WN. C, Pitch trajectory in a control bird that restores baseline pitch (black dotted line) within $5 \mathrm{~d}$ after WN is switched off (red star). Shown are daily pitch averages (black dots), the daily SDs (vertical bars), and the aversive reinforcement zone (gray-shaded area). The last baseline day is marked by a black star and day 5 after WN off by a blue star. D, E, Pitch traces of the target syllable averaged over all renditions produced on (1) the last baseline day (black); (2) the last WN day (red, cut at the onset of WN, dashed red line); and (3) the fifth day after WN off (blue). All traces are aligned to the last of the three pitch measurements (time origin). The black vertical lines delimit the interval in which pitch measurements are taken, and the black dashed lines delimit the 95th percentile of that interval relative to syllable onset. Shaded areas indicate \pm 1 SD. $\boldsymbol{E}$, Inset (gray rectangle) in $\boldsymbol{D}$ showing the increase in pitch (red) after reinforcement and the recovery thereafter (blue). Note that the bird also increased pitch outside the reinforced interval (outside the 95th percentile), revealing nonlocal effects of pitch reinforcement. $\boldsymbol{F}$, Histograms demonstrating that daily detection time jitter (mean-subtracted) was small and consistent across days (colored as in $\boldsymbol{D}, \boldsymbol{E}$ ).

eral lesions in NCM, pitch restoration is severely impeded. We show that impaired pitch restoration is not due to a deficit in motor plasticity or severe degradation of auditory processing, thus implicating NCM in recall of a pitch memory used for song maintenance.

\section{Materials and Methods}

Subjects and song recordings. Our study included 19 adult male zebra finches (Taeniopygia guttata) raised in our breeding facility. Birds were 85- $409 \mathrm{~d}$ old at the beginning of the experiments. Results from 5 additional young (70- to 75-d-old) male zebra finches were included for the assessment of auditory discrimination performance. Birds were housed individually in sound-attenuating recording chambers (IAC; inner size $60 \times 60 \times 60 \mathrm{~cm})$ on a 14/10 h day/night schedule and were given ad libitum access to food and water. After $2-3 \mathrm{~d}$ of acclimatization in the chamber most birds resumed a normal singing rate. Songs were recorded with a wall-attached microphone (Audio-Technica PRO42) at a sampling rate of $32 \mathrm{kHz}$, and were then used to train a song syllable recognizer (a two-layer neural network), which detected in real time a particular song syllable targeted for pitch reinforcement.

All the experimental procedures were in accordance with the Veterinary Office of the Canton of Zurich.

Pitch reinforcement. To selectively reinforce certain versions of a target harmonic syllable, we ran a custom LabVIEW (National Instruments) program that continuously analyzed the stream of microphone signal to detect the syllable and calculate its pitch (fundamental frequency): after syllable detection and following a fixed delay, we calculated one value of pitch every $4 \mathrm{~ms}$ (each based on the last $16 \mathrm{~ms}$ of microphone signal) using an adaptation of the Harmonic Product Spectrum algorithm (Noll, 1970). Three consecutive pitch measurements were taken and were combined to decide whether or not to play white noise (WN) through a loudspeaker (Harman/Kardon HKS 4BQ): When driving pitch up, we played $50 \mathrm{~ms} \mathrm{WN}$ acoustic stimuli when at least one measurement was below a manually chosen threshold $\theta$. Thus, to escape $\mathrm{WN}$, the minimum $p$ of the three pitch measurements had to be higher than $\theta$. Analogously, when driving pitch down, the maximum pitch value (also referred to as p) had to be smaller than $\theta$. We adjusted the threshold $\theta$ on a daily basis before lights on (i.e., before birds begin to sing) to near the middle of the previous day's pitch distribution; thereafter we kept $\theta$ constant during the day. The WN amplitude depended linearly on pitch up to $5 \mathrm{~Hz}$ below or above threshold; beyond that the $\mathrm{WN}$ amplitude was constant (independent of pitch; Fig. 1A). Note: in our birds, the $5 \mathrm{~Hz}$ linear range corresponds to approximately half the average SD of the daily pitch distribution. WN stimuli were delivered $12 \mathrm{~ms}$ after the third pitch measurement.

We counted the number of false positive and false negative syllable detections, both of which could interfere with pitch reinforcement. We found the false positive detection rate to be negligibly small, averaging $0.35 \%$ (range 0.04 to $1.64 \%, N=10$ birds). False positives were discarded in all pitch analyses. We determined the true positive rate on the last baseline day before WN playback by counting the actual number of target syllables produced by manual syllable clustering and by comparing that number to the number of correctly detected syllables, which yielded a positive detection rate of $99.4 \%$ (range 97.2 to $100 \%, N=10$ birds). Thus, the false negative detection rate was $0.6 \%$ on average.

The pitch traces within a syllable never being perfectly flat, our pitch estimates $p$ might be influenced by fluctuations of both syllable detection time and song tempo. We observed nonetheless that such possible influences had a negligible effect on our pitch estimates. We first defined the syllable detection time as the time interval between syllable onset (determined by threshold crossing of the root mean square sound waveform) and the syllable detection event reported by the syllable recognizer. Detection times exhibited a very small jitter. Their average SD was $2.1 \mathrm{~ms}$ (range 1.5 to $3.7 \mathrm{~ms}$ in $N=10$ birds, evaluated on the last day before WN playback; Fig. $1 F$ ), which is less than $1 \mathrm{~ms}$ above the lower bound dictated by aliasing errors (the latter account for $1.2 \mathrm{~ms}$ of jitter given the $4 \mathrm{~ms}$ intervals between pitch samples). In comparison, the average SD of target syllable duration was $3.1 \mathrm{~ms}$ (range 1.6 to $5.2 \mathrm{~ms}, N=10$ birds), demonstrating that the detection jitter was considerably smaller than the syllable duration jitter.

During baseline days we found a significant correlation $(p<0.01)$ between pitch and detection time in only 3 of 10 birds (with Pearson's 
correlation coefficients $-0.22,-0.14$, and -0.36 , respectively), revealing that in most animals our pitch estimates were robust to changes in detection time and song tempo. Over the course of an experiment, the syllable detection time drifted in individual animals: the $d^{\prime}$ value (see below, Data analysis) between syllable detection times during baseline singing and at the end of the experiment $(\sim 1$ month later $)$ was in the range -2.5 to $2.5(N=10$ birds, average 0.4$)$. To test for possible spurious pitch changes due to drifts in detection time, we computed the $d^{\prime}$ between baseline pitch and pitch at the end of the experiment in two different ways: with pitch measurements taken either at fixed intervals after detection time $\left(d_{d t}^{\prime}\right)$ or after syllable onset $\left(d_{o n}^{\prime}\right)$. We obtained a negligible difference $d_{d t}^{\prime}-d_{\text {on }}^{\prime}$ within the range -0.14 to $0.26(N=10$ birds, average difference 0.02). Thus, our findings did not depend on whether we measured pitch in relation to syllable detection or in relation to syllable onset.

Surgery. Birds received either bilateral NCM lesions or bilateral sham lesions. Before surgery, birds were anesthetized with isoflurane and head fixed in a stereotaxic frame. Their head was set to an angle of 65 degrees from horizontal, measured at the anterior surface of the skull. To perform the lesions, we vertically injected $500 \mathrm{nl}$ of ibotenic acid (SigmaAldrich I2765) solution in ddH2O $(7 \mathrm{mg} / \mathrm{ml})$ in each hemisphere through a glass pipette (two injections per penetration, one at $1500 \mu \mathrm{m}$ ventral from the surface of the brain, the other at 2000-2250 $\mu \mathrm{m}$ from the surface). For sham lesions, we similarly injected $500 \mathrm{nl}$ of Ringer's solution in each hemisphere. All injections were made at a lateral distance from the midline of about $500 \mu \mathrm{m}$ (lesioned birds: average $460 \mu \mathrm{m}$, range 340 to $520 \mu \mathrm{m}, N=6$ birds; sham-lesioned birds: average $490 \mu \mathrm{m}$, range 350 to $650 \mu \mathrm{m}, N=4$ birds) and about $800 \mu \mathrm{m}$ anterior of the bifurcation of the mid-sagittal sinus (lesioned birds: average $807 \mu \mathrm{m}$, range 650 to $1000 \mu \mathrm{m}, N=6$ birds; sham-lesioned birds: average $773 \mu \mathrm{m}$, range 710 to $810 \mu \mathrm{m}, N=4$ birds). The craniotomy was then sealed with Kwik-Cast (World Precision Instruments) and the scalp was closed with veterinary glue (GLUture; Abbott Laboratories). Birds typically resumed singing in the evening or the morning after surgery.

Song restoration. After surgery, no more WN playbacks were delivered. Based on visual inspection of the spectrograms, the first songs produced after surgery were not visually different from presurgery songs (Fig. $4 F$ ), which led us to conclude that lesions did not have major immediate effects on vocal output. We used the daily singing rate to evaluate the birds' well being. The fraction of target syllables sung postsurgery relative to presurgery quickly approached $1(0.41$ on the first recovery day, range 0.04 to $1.53, N=10$ birds; and 0.98 on the second recovery day, range 0.41 to $1.86, N=10$ birds).

The target syllable pitch was monitored on a daily basis and its restoration was assessed relative to pitch on the last baseline day before $\mathrm{WN}$ playbacks.

Data analysis. For any pair of different days $i$ and $j$, we compared syllable properties such as pitch, entropy, or detection time through the $d^{\prime}$ measure that weighs differences between averages by the underlying variances:

$$
d_{i, j}^{\prime}=\frac{\sqrt{2}\left(\mu_{i}-\mu_{j}\right)}{\sqrt{\sigma_{i}^{2}+\sigma_{j}^{2}}},
$$

where $\mu_{i}$ is the average value on day $i$ and $\sigma_{i}^{2}$ its variance. In the case of equal variances $\left(\sigma_{i}^{2}=\sigma_{j}^{2}\right), d_{i, j}^{\prime}$ reports the difference in average between the two days in the convenient unit of SDs.

To determine whether the daily average pitch significantly deviated from baseline pitch, we estimated a pitch significance threshold as follows. We analyzed song recordings from six birds that were not subjected to surgery or pitch reinforcement and that produced a complex syllable similar to the target syllable in Figure $1 B$. We first estimated the variability of daily average pitch in these birds. That is, we collected the daily average pitch $\bar{p}_{i}$ and SD $\sigma_{i}$ on 7 consecutive days $i=1, \ldots, 7$. We then computed the mean baseline pitch $\bar{p}_{b}=\left\langle\bar{p}_{i}\right\rangle_{i}$ and subtracted it from the daily average pitch, which after normalization by the $\mathrm{SD} \sigma_{i}$ resulted in the following daily pitch $z$-score:

$$
Z_{i}=\frac{\bar{p}_{i}-\bar{p}_{b}}{\sigma_{i}}
$$

We then considered all $z$-score values from the six different birds to yield 36 independent $z$-score estimates (42 estimates minus 6 degrees of freedom from subtraction of the averages). The SD of these $z$-score estimates was 0.29 . To achieve a significant deviation of $z$-score at the $1 \%$ level, the daily $z$-score must be at least 2.58 times larger than this value, corresponding to $0.29 \times 2.58=0.75$. Thus, the significance threshold for pitch deviations is 0.75 : on a day $i$, a bird significantly changed the average pitch away from baseline when the $z$-score $Z_{i}$ was higher than 0.75 .

To assess significance of pitch difference on a day $i$ relative to the last baseline day $b$ before $\mathrm{WN}$ playback, we tested whether the absolute value $\left|d_{i, b}^{\prime}\right|$ exceeded a threshold value of $d_{\theta}^{\prime}=0.75$. This measure is therefore an evaluation of significance of pitch change relative to the naturally occurring variability of baseline pitch.

Based on our observations in control birds (see Results), we analyzed pitch restoration in a "late window" from 6 to $20 \mathrm{~d}$ from the first day without $\mathrm{WN}$. We quantified the amount of pitch recovery on day $i$ after end of WN by computing the residual pitch $D_{i}$ defined as the normalized difference between mean baseline pitch $\bar{p}_{b}$ and mean daily pitch $\bar{p}_{i}$ on that day, normalized to the difference between $\bar{p}_{b}$ and mean daily pitch $\bar{p}_{0}$ on the last day with $\mathrm{WN}$ :

$$
D_{i}=\frac{\bar{p}_{i}-\bar{p}_{b}}{\bar{p}_{0}-\bar{p}_{b}}, \quad i=1,2,3, \ldots
$$

We assessed pitch recovery in the late window in terms of the mean residual pitch

$$
D_{L}=\left\langle D_{i}\right\rangle_{i}
$$

across days $i$ and its $\mathrm{SD} \sigma_{L}$. A mean $D_{L}$ near zero indicates recovery of baseline pitch, whereas a small SD $\sigma_{L}$ indicates stable pitch.

To assess pitch stability also in two control birds that were subjected to NCM lesions but not to pitch reinforcement (for these birds $D_{i}$ is undetermined because $\bar{p}_{0}=\bar{p}_{b}$ ), we assessed pitch stability (in the late window) in terms of absolute $d^{\prime}$ values. In particular, we computed the late pitch deviation $B$ from baseline by averaging:

$$
B=\left\langle\left|d_{i, b}^{\prime}\right|\right\rangle_{i}
$$

with $i$ ranging across all days in the late window and $b$ the last baseline day without WN (or the last day before surgery for birds without pitch reinforcement). $B$ close to zero indicates both highly similar and highly stable pitch, because the absolute value guarantees that pitch fluctuations near zero do not average out. In contrast, when $B$ is far from zero, which is what we observe in NCM-lesioned birds, the pitch in the late window could either be dissimilar to baseline pitch or unstable. To test for the latter, we assessed the pitch fluctuation $F$ in the late window by computing the average $d^{\prime}$ between all days $i$ and $j$ in the late window:

$$
F=\left\langle\left|d_{i, j}^{\prime}\right|\right\rangle_{i \neq j} .
$$

To assess stability of the produced song after NCM lesions, we analyzed the target syllable structure in terms of Wiener entropy, a measure of "tonality" that ranges from minus infinity (pure tones) to zero (white noise; Tchernichovski et al., 2000). We computed the Wiener Entropy of $16 \mathrm{~ms}$ sound snippets in steps of $4 \mathrm{~ms}$ using a custom MATLAB (MathWorks) script. For each bird, we first averaged entropy values over the duration of the syllable and then over all syllable renditions on 1 day, shown in Figure $4 E$.

Auditory discrimination. To test whether bilateral NCM lesions impaired the birds' hearing, we subjected 8 birds (26-38 d after lesions) to a simple auditory discrimination experiment based on an operant conditioning paradigm presented in Tokarev and Tchernichovski (2014). The experimental setup is described in Figure $4 A$ and $B$. A male bird (Fig. $4 A$, in the foreground) was housed individually in a cage containing a small window from which he could see a female zebra finch in a neighboring cage inside the same recording chamber. Near the window we placed a perch that was the only vantage point from which the bird could 
see the female. The female's presence attracted the bird onto the perch, which was built as a lever that triggered an electric contact as soon as the bird sat on it.

All trials began after the bird had been sitting on the perch and not vocalizing for at least $2-3 \mathrm{~s}$ (Fig. $4 B$, bird-on-perch probability 1 ). At trial onset (time 0 ) we randomly (with $50 \%$ probability) played one of two different song bouts (from two different zebra finches) through a loudspeaker. One of the songs was always followed by a $1 \mathrm{~s}$ air puff starting $3 \mathrm{~s}$ after playback onset. The puff tended to blow the bird off the perch (Fig. $4 B$, sudden drop of the black curve at $3 \mathrm{~s}$ ), thus providing a mild aversive reinforcement signal (the cage was large enough for the bird not to get hurt). The other song was not paired with an air puff.

After a brief training period (see Results), birds began to increasingly leave the perch before air puff onset to avoid the air puffs. Most importantly, they left the perch more often during the song paired with an air puff than during the other song (Fig. 4C). To evaluate song discrimination in these experiments, we calculated (at $4 \mathrm{~ms}$ time resolution) the conditional perch probabilities $P$ (perch $\mid$ puff) and $P$ (perch|nopuff) that the bird sat on the perch as a function of time since trial onset. We calculated these probabilities both for trials of puffed and of nonpuffed songs (Fig. 4B). We then compared these probabilities using a difference between proportions significance test: in every $4 \mathrm{~ms}$ time bin we first calculated the pooled sample proportion as follows:

$$
p s p=\frac{n_{\text {perch|puff }}+n_{\text {perch|puff }}}{n_{\text {puff }}+n_{\text {nopuff }}},
$$

where $n_{\text {puff }}$ and $n_{\text {nopuff }}$ are the numbers of trials with and without air puffs, and $n_{\text {perch|puff }}$ and $n_{\text {perch|nopuff }}$ are the number of puff/no puff trials in which the bird was on the perch during that time bin. The standard error of the sample proportion is then

$$
S E=\sqrt{\frac{p s p(1-p s p)}{n_{p u f f}}+\frac{p s p(1-p s p)}{n_{\text {nopuff }}}},
$$

based on which we calculated the difference ( $z$-score)

$$
Z=\frac{P(\operatorname{perch} \mid \text { puff })-P(\operatorname{perch} \mid \text { nopuff })}{S E},
$$

with $P($ perch $\mid$ puff $)=\frac{n_{\text {perch } \mid \text { puff }}}{n_{\text {puff }}}$ and $P($ perch $\mid$ nopuff $)=\frac{n_{\text {perch } \mid \text { nopuff }}}{n_{\text {nopuff }}}$. The $p$ value associated with that difference is given by the normal cumulative distribution as follows:

$$
p(Z)=\sqrt{\frac{2}{\pi}} \int_{-\infty}^{Z} e^{-t^{2} / 2} d t .
$$

After some training, all birds reached a significant difference $(p<0.01)$ between $P($ perch $\mid$ puff $)$ and $P($ perch $\mid$ nopuff $)$ within less than $3 \mathrm{~s}$ after playback onset (that is, before the air puff trigger, see Results), thus demonstrating an ability to discriminate sounds, which is a necessary requirement for song maintenance.

Histology. At the end of the experiments, lesioned birds were deeply anesthetized with an overdose of pentobarbital sodium and perfused via the left ventricle with a $4 \%$ paraformaldehyde solution (P6148; SigmaAldrich). The brains were removed and fixed in the same solution overnight. Sagittal sections ( $80-100 \mu \mathrm{m}$ thickness) were cut and Nissl stained with cresyl violet to highlight cell bodies. For each hemisphere, we selected the brain slice showing the largest lesioned surface area and isometrically aligned it to a previously drawn outline of the songbird auditory lobule extracted from a sagittal reference section about $500 \mu \mathrm{m}$ from the midline (Fig. 2C). As landmarks for alignment we chose the ventricle $(\mathrm{V})$ that separates the nidopallium from the hyperpallium and the lamina mesopallialis ( $\mathrm{LaM}$ ), which separates the nidopallium from the mesopallium. In each aligned brain slice we drew a dot at the approximate center of the lesioned area (Fig. 2C). Injection sites in different birds were then compared based on their positions relative to the brain outline (Fig. 2D). The NCM region is broadly defined as the most posterior part of the medial nidopallium (Fig. $2 D$, yellow area).

Because lesion damage in experimental birds was assessed only 3 weeks or more after surgery, we evaluated the extent of the lesions at different time points in four additional birds. Lesions were made as described above, and birds were killed 1, 7, 14, and $32 \mathrm{~d}$ later. From the bird perfused $1 \mathrm{~d}$ after surgery we estimated the initial lesioned volume to extend about $640 \mu \mathrm{m}$ laterally from the injection site (eight sections each $80 \mu \mathrm{m}$ thick), and about $430 \mu \mathrm{m}$ anterior from the injection site (measured in the slice showing the largest lesioned area; Fig. 2A). Over time, possibly due to neurogenesis and cell migration, the lesioned areas gradually shrunk due to the influx of cells with large cell bodies (Figs. $2 A, B$ ). Note that the exponential fit in Figure $2 B$ only provides a qualitative description of lesion size development; we are not suggesting a predictive model of lesion size with a fixed time constant. Given this shrinkage, histology from experimental birds could not reveal the original extent of the lesioned regions-only their approximate location.

All measurements were taken with NIH ImageJ.

\section{Results}

We subjected adult male zebra finches to a pitch reinforcement paradigm (Tumer and Brainard, 2007; Andalman and Fee, 2009; Warren et al., 2011) in which a WN acoustic stimulus is played back every time the pitch of a target syllable produced is lower (respectively, higher) than a certain threshold value. As a result, birds tend to raise (respectively, lower) the pitch of the target syllable to escape WN. Typically, when WN playback is ceased, birds spontaneously restore baseline pitch levels within the course of several days (Tumer and Brainard, 2007; Warren et al., 2011). Successful song restoration indicates that, during pitch shifting, birds maintain a memory of a target song, which could be either a memory of the tutor song that birds acquired during a sensory-learning phase (Immelmann, 1969; Zann, 1996) or a memory of their recently produced song. Thus, this pitch restoration process enables us to experimentally evaluate the availability of a song memory and/or its involvement in shaping vocal motor output.

We assessed pitch changes using the $d^{\prime}$ measure that expresses pitch differences in units of SDs (see Materials and Methods). In each bird, after recording baseline (unperturbed) singing, we reinforced the pitch of a target syllable containing a harmonic stack. We repeatedly adjusted the pitch threshold for $\mathrm{WN}$ playbacks to drive highly significant pitch increases/decreases: at the end of the pitch reinforcement phase, the absolute pitch change relative to baseline was on average $d^{\prime}=3.3$ (range 2.2 to $5.2, N=10$ birds). The pitch reinforcement phase lasted on average $11 \mathrm{~d}$ (range 6 to $15 \mathrm{~d}, N=10$ birds), depending both on the birds' performance and on how often and how much we changed the threshold for WN playback. After pitch had been sufficiently shifted, we ended WN playbacks to release birds from pitch reinforcement. Birds were divided into two groups: a first group that received bilateral neurotoxic NCM lesions and a second group that received bilateral sham lesions. Right after neurotoxic and sham lesions, birds were released from pitch reinforcement.

\section{Sham-lesioned control birds successfully restore baseline pitch}

Control birds receiving sham lesions changed their pitch on average by a $d^{\prime}=3.9$ relative to baseline (range 3.3 to $5.2, N=4$ birds) within on average $10.3 \mathrm{~d}$ (range 9 to $11 \mathrm{~d}$ ). After sham NCM lesions (made via saline injections) they readily recovered their baseline pitch: the $d^{\prime}$ to baseline pitch fell below 0.75 (corresponding to our estimated $1 \%$ significance threshold level; see Materials and Methods) on average on the sixth day after WN end 
A

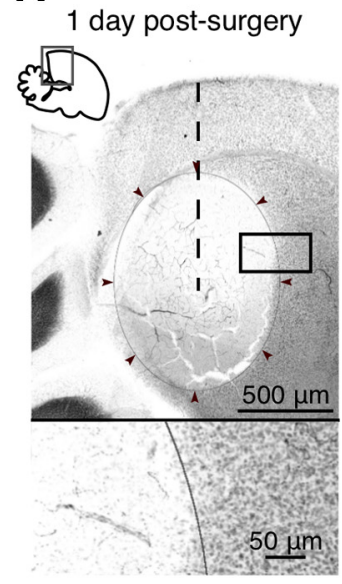

7 days post-surgery

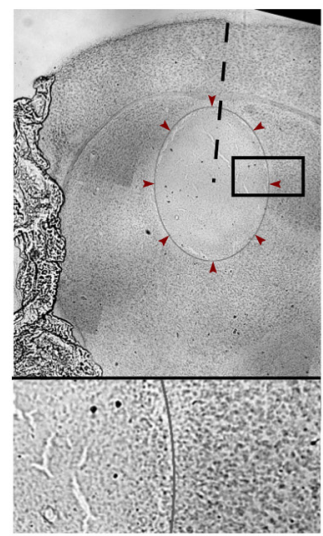

14 days post-surgery

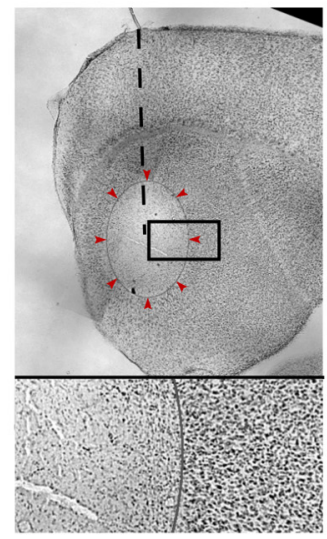

32 days post-surgery

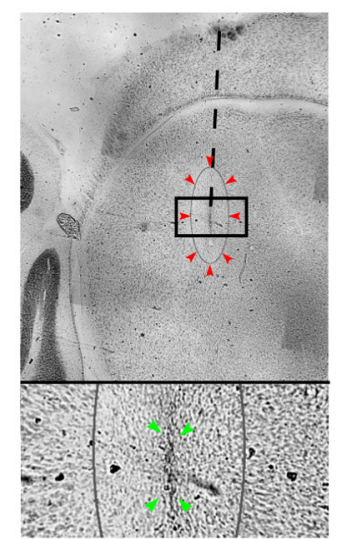

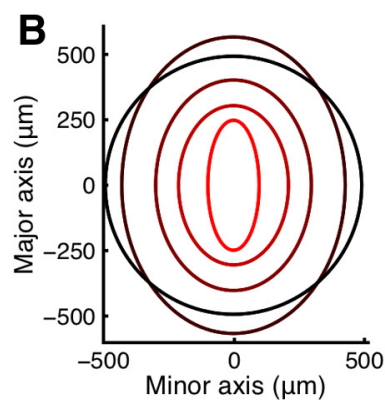
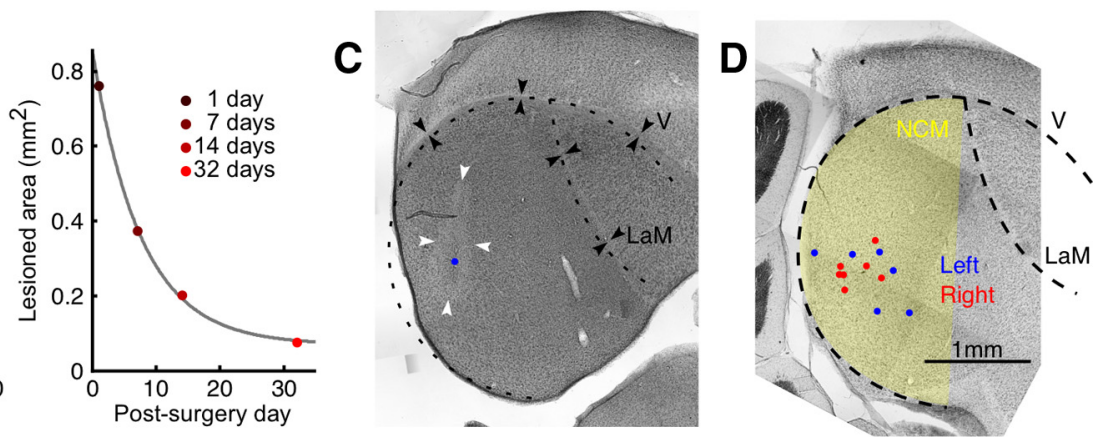

Figure 2. Histology demonstrating NCM lesions. $A$, Nissl-stained sagittal brain sections from four birds killed 1,7, 14, and $32 \mathrm{~d}$ (from left to right) after ibotenic acid injections into NCM (these sections were taken from birds that did not participate in the experiment). The lesioned areas (delimited by gray ellipses and colored arrowheads) are defined by absence of large cell bodies. Dashed lines indicate the approximate paths of the injection pipettes. Bottom, Zoomed in view of the area inside the rectangles on top. On day 32 after surgery (rightmost) a glial scar is clearly visible in the middle of the rectangle (green arrowheads). $\boldsymbol{B}$, Left, Overlay of the ellipses in $\boldsymbol{A}$ (with colors matching the arrowhead colors in $\boldsymbol{A}$ ). As a reference, we also drew a black circle of radius corresponding to a $500 \mathrm{nl}$ sphere (injected acid volume). Right, Ellipse area (colored dots) as a function of postsurgery survival time, shown together with an exponential fit (gray curve). C, Example sagittal brain section of a bird perfused $28 \mathrm{~d}$ after ibotenic acid injection, showing the fits (dashed lines) to the ventricle $(V)$ and the LaM (black arrowheads). The estimated injection site is marked by a blue dot. White arrowheads indicate the area where tissue damage is still visible. $\boldsymbol{D}$, Estimated injection sites (blue dots, left hemisphere; red dots, right hemisphere) for all NCM-lesioned birds (six were pitch reinforced, two were not). For three hemispheres we were unable to establish the injection site. The NCM region is highlighted in yellow.

(range 3 to $9 \mathrm{~d}, N=4$ birds). In addition, after falling below threshold level, the pitch was stable and did not show large fluctuations (Fig. 3).

Based on these observations we defined an early recovery window (days 1-5 after $\mathrm{WN}$ end) within which control birds recovered baseline pitch and a late recovery window (days 6-20 after $\mathrm{WN}$ end) within which recovered pitch was on average stable (see also Materials and Methods). To assess the level of pitch recovery in the late window, we computed the average pitch deviation $B$ from pitch on the last baseline day (before WN playback, pitch deviation is reported in terms of absolute $d^{\prime}$ values; see Materials and Methods). Across all sham-lesioned birds the pitch deviation to baseline was $B=0.46 \pm 0.21$ (range 0.25 to $0.72, N=4$ birds), revealing that pitch had recovered within the early window and was then maintained stably during the late window. In these birds, the average pitch fluctuation $F$ (absolute $d^{\prime}$ between late recovery days; see Materials and Methods) of daily pitch during the late recovery window was $F=0.27 \pm 0.11$ (range 0.13 to 0.36 , $N=4$ birds), revealing that pitch fluctuations were small after pitch had recovered. We obtained similar results when we evaluated the average residual pitch $D_{L}$ across the late recovery window, which is a measure that compensates for differences in the magnitude of reinforced pitch changes. We found that the residual pitch in the late window was on average $D_{L}=0.04 \pm 0.12$ (range -0.08 to $0.16, N=4$ birds) and had an SD of $\sigma_{\mathrm{L}}=0.05 \pm$
0.02 (range 0.03 to $0.08, N=4$ birds), revealing both small deviation from baseline pitch and small variability (Fig. 3).

\section{NCM lesions impede song restoration}

We drove the pitch in birds to receive NCM lesions by on average $d^{\prime}=2.9$ from baseline pitch (range 2.2 to $4.2, N=6$ birds) within on average $11.3 \mathrm{~d}$ (range 6 to $15 \mathrm{~d}, N=6$ birds). After WN end and bilateral NCM lesions, birds failed to restore pitch as quickly and consistently as control birds did (Fig. 3). Across all NCMlesioned birds, the average pitch deviation $B$ away from baseline in the late window was $B=1.45 \pm 0.64$ (range 0.56 to $2.29, N=$ 6 birds), revealing significantly less recovery than in shamlesioned birds (Student's $t$ test, $p=0.02$ ). The average pitch fluctuation in the late window was $F=0.70 \pm 0.33$ (range 0.22 to $1.19, N=6$ birds), revealing significantly larger pitch variability in NCM-lesioned birds compared with controls (Student's $t$ test, $p=0.04)$. In NCM-lesioned birds the residual pitch $D_{L}$ and its SD $\sigma_{L}$ in the late window were $D_{L}=0.28 \pm 0.54$ (range -0.54 to $0.85, N=6$ birds) and $\sigma_{L}=0.23 \pm 0.14$ (range 0.07 to $0.41, N=$ 6 birds), which is, respectively, 7 and 4.6 times larger than in sham-lesioned birds (Fig. 3). In one bird we monitored pitch until $45 \mathrm{~d}$ after surgery, at which time pitch was still far away from baseline (normalized residual pitch $D_{i}=-0.55$ ) and was strongly fluctuating. 


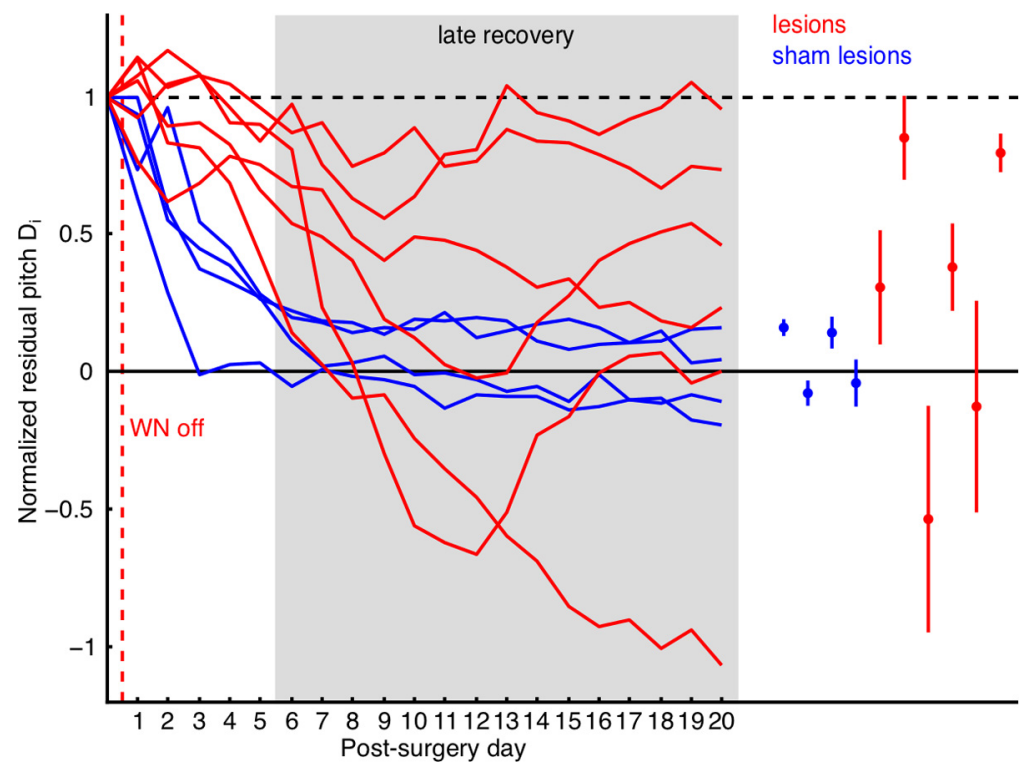

Figure 3. NCM lesions impair pitch restoration. Left, Four sham-lesioned birds exhibit pitch recovery trajectories (blue curves) that quickly converge toward baseline pitch (zero residual pitch), whereas trajectories in six NCM-lesioned birds do not converge (red curves). Residual pitch trajectories are normalized such that 1 (dashed black line) corresponds to pitch on the last WN day and 0 corresponds to baseline pitch. The 15 late recovery days are marked by a gray-shaded area, and the WN off event by a red dashed line. Right, In sham-lesioned birds the late-recovery residuals both are closer to zero (blue dots correspond to late-recovery averages in individual birds) and fluctuate less than corresponding residuals in NCM-lesioned birds (red dots; vertical bars indicate $\pm 1 S D$ of late recovery residuals).

\section{NCM-lesioned birds tend to revert pitch in the direction of baseline}

Despite not restoring baseline pitch like sham-lesioned birds did, each of the six birds with NCM lesions showed a tendency of changing pitch toward baseline (Fig. 3), which argues against a complete loss of target pitch memory. We tested whether the postlesion decrease in daily pitch deviation from baseline was consistent with random pitch drift: across all lesioned birds the median $d^{\prime}$ (1.33, $N=90 d^{\prime}$ values) between late-window and baseline pitches was significantly different from the median $d^{\prime}$ (2.64, $N=6 d^{\prime}$ values, $p=1.4 \times 10^{-4}$, Wilcoxon rank-sum test) between pitches on the first recovery day and baseline, revealing that birds significantly changed pitch over the course of recovery.

We also tested whether random walks could explain observed $d^{\prime}$ trajectories drifting toward baseline by calculating the probability that six (the number of birds) 20-step (the length of the full recovery window) random walks with equal up and down probabilities in each step remain on the negative side between steps 6 and 20 (the late window). From a sample of $10^{9}$ sets composed of six random walks each, only a fraction $p=1.7 \times 10^{-4}$ remained exclusively on the negative side in the "late window," implying that our results are highly unlikely to be explained by balanced random pitch drift and that post-NCM-lesion paths indeed show nonzero drift toward baseline.

\section{NCM lesions do not destabilize pitch}

To test whether the large pitch fluctuations observed after NCM lesions were due to the preceding pitch reinforcement, we made bilateral lesions in two birds that were never pitch reinforced before. After surgery both birds maintained a stable pitch for up to $20 \mathrm{~d}$; the pitch fluctuations $F$ in the late window were $F=0.15$ and 0.31 , respectively. Also, postlesion pitch was not notably different from prelesion pitch. The average pitch deviation between the day before the lesion and all late recovery days was $B=0.17$, respectively, 0.43 . Thus, the amount of pitch drift and fluctuations caused by NCM lesions is no larger than that in sham-lesioned (and pitch reinforced) birds. We conclude that NCM lesions alone do not explain the loss of pitch stability; they do so only in a state of reinforced pitch away from baseline.

\section{NCM lesions do not prevent successful escaping from aversive reinforcement} To evaluate the effect of lesions on processing of auditory feedback and motor plasticity, in four birds we tested whether they could escape aversive reinforcement after receiving large bilateral NCM lesions. Before the lesions we briefly verified that birds were able to significantly drive pitch away from baseline. Then, after letting birds restore baseline pitch, we made large bilateral NCM lesions and attempted to reinforce pitch again 3, 10, 19, and $20 \mathrm{~d}$ after the lesions. All birds successfully changed pitch in the expected direction (Fig. 4D). After only $4 \mathrm{~d}$ of $\mathrm{WN}$ playbacks, birds had changed their pitch from baseline to a $d^{\prime}$ of $2.66 \pm 0.43$ (range 2.10 to $3.14, N=4$ ). This reveals that NCM lesions do not affect birds' ability to selectively change their motor program.

\section{NCM lesions do not affect syllable entropy}

Visual inspections of song spectrograms (Fig. $4 F$ ) as well as pitch measurements at baseline suggest that NCM lesions have no major impact on song production. We further evaluated the stability of the target syllable by tracking its daily mean Wiener entropy. In case of song degradation we would expect entropy values to become less negative (i.e., syllables become noisier). In lesioned birds the syllable entropy did not consistently increase between the last baseline day before $\mathrm{WN}$ and the last day of the late recovery window (Fig. $4 E$ ): the $d^{\prime}$ between average entropy on these 2 days was in the range -0.50 to 0.94 (mean $0.24 \pm 0.51$, $N=6$ birds, positive $d^{\prime}$ values indicating increased entropy, hence noisier syllable). Thus, NCM lesions did not impact the spectral noise of the target syllable that may have interfered with pitch restoration.

\section{NCM lesions do not severely impair hearing}

Aversive reinforcement is not well suited to study the ability to process natural auditory stimuli, because escape from $\mathrm{WN}$ can be achieved by correlating the presence/absence of WN with motor variability (instead of the auditory feedback thereof). We thus tested whether NCM lesions cause impairments of auditory discrimination that may be underlying the evaluation of pitch feedback during song restoration. We made NCM lesions in eight birds (three adults and five young birds) before subjecting them to an air-puff song discrimination task. In this task, birds are exposed to two auditory stimuli, one of which is always followed by an air puff. Birds that are able to discriminate between the stimuli escape from the perch more often during the air puffpredicting (negatively reinforced) stimulus than during the other stimulus (see Materials and Methods). 


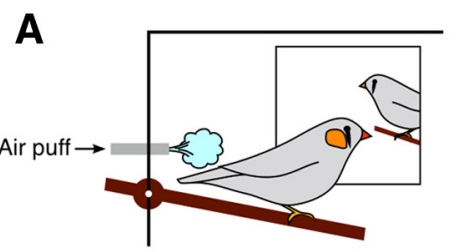

C

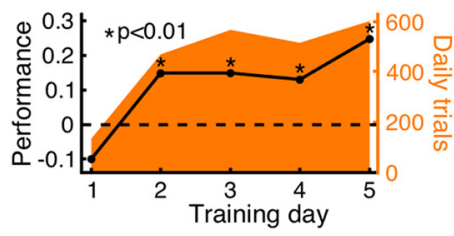

D

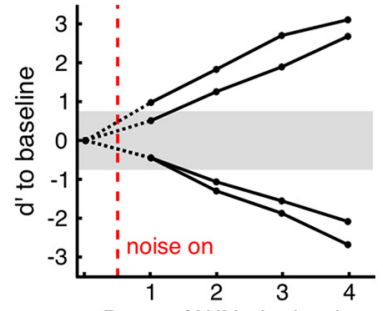

Days of WN playback

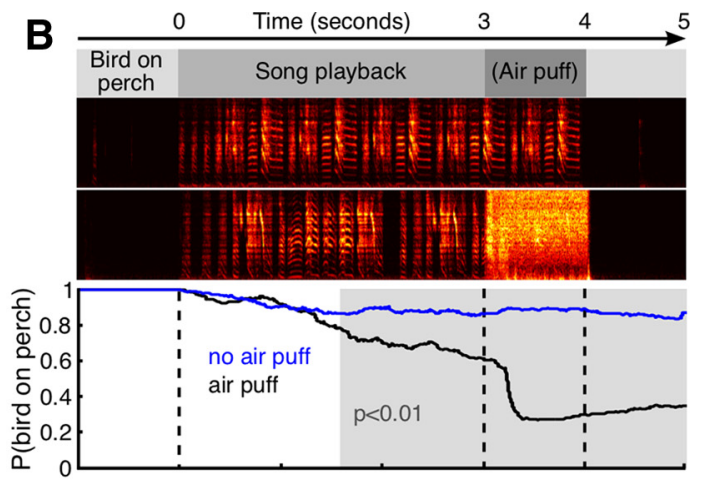

E

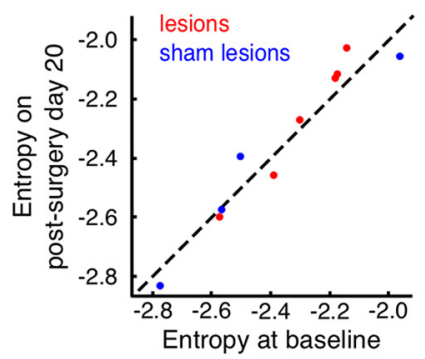

Bird 3

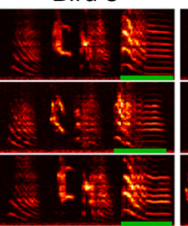

Bird 4

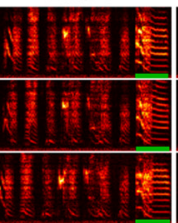

Bird 5

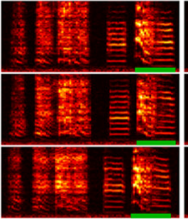

Bird 6

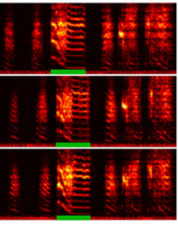

Figure 4. NCM-lesioned birds show no deficit in auditory and motor performance. $\boldsymbol{A}$, Schematic of the song discrimination setup. During trial sessions, when the experimental bird (in the foreground) sits on the perch, the computer triggers random playback of one of two songs, one of which is followed by an air puff coming from a tube just next to the perch. $\boldsymbol{B}$, An NCM-lesioned bird successfully discriminates the two songs (shown are data from the fifth day of training; Bird A1 in Table 1). Below the time line (top), two song spectrograms show the nonpuffed song (upper) and the puffed song (lower, with air puff noise visible between 3 and 4 s into the trial). Bottom plot, The bird-on-perch probability as a function of time in the trial (daily performance of 600 trials) shows that on trials without air puffs the bird remains throughout on the perch in more than $80 \%$ of the trials (blue curve), whereas on air puff trials the bird stays on the perch for $3 \mathrm{sin}$ only about $60 \%$ of cases (black curve). The significant difference between black and blue curves ( $p<0.01$, gray background) before air puff onset indicates that the bird is able to discriminate between the two songs. C, Consistent auditory discrimination performance over consecutive days in one example bird (same bird as in $\boldsymbol{B}$ ). We report the performance as probability difference $P($ perch $\mid$ nopuff $)-P($ perch $\mid$ puff $)$ of the bird being on the perch at the $3 \mathrm{~s}$ mark on trials without and with air puff (black line), together with the number of trials done on each day (orange). The low number of playbacks on the first training day is often due to the bird getting used to the air puff and not quickly returning on the perch for more trials. From the second training day the bird's performance is positive and significant (black stars: $p<0.01$ ). $D$, NCM-lesioned birds are able to modify pitch to avoid WN. Shown are $d^{\prime}$ pitch changes from baseline (black curves) for two birds that received bilateral NCM lesions 19 and $20 \mathrm{~d}$ before WN onset (shifted up) and for two birds that received lesions 3 and $10 \mathrm{~d}$ earlier (shifted down). All birds quickly changed pitch beyond the $1 \%$ significance range, gray-shaded rectangle). $\boldsymbol{E}$, Scatter plot (one dot per bird) of Wiener Entropy averaged over all renditions of the target syllable on the last baseline day (horizontal axis) and on postlesion day 20 (vertical axis). Blue, sham-lesioned birds; red, NCM-lesioned birds. $\boldsymbol{F}$, No motor deficit following NCM lesions is revealed by visual inspection of song motif spectrograms $(0-8 \mathrm{kHz})$ on baseline days, on postlesion day 1 , and on postlesion day $20(N=6$ birds from left to right). Green bars highlight target syllables.

All NCM-lesioned birds tested succeeded in showing a significantly higher escape rate during playback of the aversively reinforced songs. The probability that birds stayed on the perch just before the onset of the air puff dropped significantly $(p<0.01$; see Materials and Methods) after 1-4 d of practice (mean 2.4, $N=8$ birds). We quantified successful escapes by counting the trials in which a bird had left the perch at the moment we delivered the air puff (typically about 300-900 trials per day; see Table 1 for results on the last day of testing).

In conclusion, auditory processing was not significantly impacted in NCM-lesioned animals, suggesting that the impairment of pitch restoration after NCM lesions is not a result of lost auditory processing ability, but rather a remote memory involvement of NCM.

\section{Discussion}

We performed large lesions in a high auditory area of the songbird brain and studied their effects on reversing changes in adult song. Lesions impaired the restoration of baseline pitch but had no noticeable effects on learning of an auditory discrimination task. Postlesion songs were indistinguishable from prelesion songs, strengthening previous reports on smaller NCM lesions (about one-fifth of our injected neurotoxin volumes) preserving song stability (Gobes and Bolhuis, 2007). Furthermore, lesions spared successful pitch reinforcement, revealing that a fully intact NCM is not required for mediating selective pitch changes. The lesions we made targeted the most posterior/medial part of the nidopallium, including regions in which stimulus-specific neural response habituation was observed in multiunit recordings (Chew et al., 1995) and in which protein synthesis blockage in juveniles lead to impaired tutor song imitation (London and Clayton, 2008). The mediolateral extent of our lesions was so large that it also covered more lateral parts of NCM where immediate early gene expression correlates with song learning (Bolhuis et al., 2000) and where lesions reduce behavioral preference for 
Table 1. Auditory discrimination in NCM-lesioned birds

\begin{tabular}{lllllll}
\hline Bird & Day & $P($ perch $\mid$ nopuff $)$ & $P($ perch $\mid$ puff $)$ & $n_{\text {nopuff }}$ & $n_{\text {puff }}$ & $p$ \\
\hline A1 & 27 & 0.86 & 0.61 & 301 & 299 & $4.9 \times 10^{-12}$ \\
A2 & 35 & 0.84 & 0.65 & 308 & 292 & $4.5 \times 10^{-08}$ \\
A3 & 27 & 0.65 & 0.26 & 300 & 277 & $2.6 \times 10^{-21}$ \\
Y1 & 37 & 0.38 & 0.21 & 293 & 307 & $1.9 \times 10^{-06}$ \\
Y2 & 35 & 0.81 & 0.63 & 139 & 161 & $1.0 \times 10^{-03}$ \\
Y3 & 38 & 0.72 & 0.28 & 130 & 168 & $7.9 \times 10^{-14}$ \\
Y4 & 38 & 0.47 & 0.19 & 195 & 202 & $1.7 \times 10^{-09}$ \\
Y5 & 34 & 0.54 & 0.37 & 145 & 155 & $2.1 \times 10^{-03}$ \\
\hline
\end{tabular}

NCM-lesioned birds perform well in an auditory song discrimination task. Birds $\mathrm{A} 1-\mathrm{A} 3$ are adults, $\mathrm{Y} 1-\mathrm{Y} 5$ are young (70-75 d post hatch). Shown are the dates (the number of days after surgery) when the shown results were collected, the bird-on-perch probabilities $3 \mathrm{~s}$ into trials without and with air puffs, the numbers of trials performed on that day, and the $p$ values indicating significantly different on-perch probabilities (successful discrimination) when $p<0.01$ (see Materials and Methods).

tutor song over other songs (Gobes and Bolhuis, 2007). The extent of our applied lesions thus provides strong evidence that NCM has little, if any, role in motor performance and motor plasticity, and possibly also in feedback evaluation. Based on these results, we reject the hypothesis that motor or auditory functions relevant to pitch restoration might be responsible for the observed impairment induced by the lesions. Instead we suggest that the lesions may have damaged neural tissue necessary for either storing a memory of target pitch or for retrieving such a memory.

Our experiments do not allow us to unambiguously identify which properties of memory may have been affected. A common confound, which should be the target of further research, is the often overlooked distinction between memory storage and retrieval (Squire, 2006). Memory performance in humans assessed with forced-choice recognition is good even with probed lists of thousands of items (Standing, 1973), suggesting that recall is limited by the retrieval process rather than actual forgetting (Romani et al., 2013). A similar limitation of recall is also demonstrated by retrogradely amnesic patients who appear to retain memories but struggle to recall them (Lewis et al., 1968). The memory recall deficit in case of retrograde amnesia also has been extensively investigated in animals. Typical such studies require animals to first learn a task before they receive electrical or chemical convulsing shocks to induce retrograde amnesia. After exhibiting worsened performance, animals are given one more shock-free "reminder" trial, which is often sufficient to restore good performance (Lewis et al., 1968; Cherkin, 1972). Hence, one avenue toward testing for impairment of memory recall in songbirds could be to play back the target song to an NCM-lesioned bird that failed to recover the prelesion pitch. If lack of pitch restoration is due to inaccessibility of the target memory but not to loss of "desire" to sing a target, then birds might be able to recognize their target song from the playback and use the retrieved memory for successfully restoring their song. While such experiments could be worth conducting, it is also worth remembering that to date there are no reports that adult songbirds can form new auditory target memories past their critical sensory learning phase (Immelmann, 1969).

Our findings agree with a broad literature that ascribes a role in target song memorization to NCM (Bolhuis and Gahr, 2006; Pinaud and Terleph, 2008; Hahnloser and Kotowicz, 2010). If that target is indeed the tutor song as previously suggested (Terpstra et al., 2004), then we are drawn to conclude that pitch restoration is a sensory-guided song-learning mechanism reminiscent of developmental song learning in young birds. If, instead, NCM holds part of the memory of the bird's own song, then the im- paired pitch restoration in NCM-lesioned birds could indicate the extent to which birds rely on a memory of their own song (rather than the tutor song) for pitch restoration. Possibly, NCM lesions in pitch-reinforced isolate (nontutored) birds could help to resolve these contrasting views, though the feasibility of pitchreinforcing the highly variable songs of isolates (Immelmann, 1969; Fehér et al., 2009) is unclear.

In addition to NCM, song maintenance also relies on a basal ganglia network including the songbird striatal/pallidal nucleus Area $\mathrm{X}$ and the lateral magnocellular nucleus of the anterior nidopallium (LMAN), both of which are required for successful pitch reinforcement in adults (Scharff and Nottebohm, 1991; Kao et al., 2005; Ali et al., 2013; Kojima et al., 2013). Because Area $\mathrm{X}$ and LMAN-lesioned birds are unable to change pitch to avoid aversive reinforcement, we conclude that NCM is more selectively involved in song maintenance than are Area X and LMAN. The adult role of the latter areas seems to be the mediation of directed song changes in general, whereas the role of NCM seems to be to support song maintenance in particular. For a particular song feature (pitch), we thus identified an anatomical difference between a natural learning process (spontaneous restoration of baseline pitch) and an externally enforced process (pitch shift to escape WN). While both processes rely on LMAN and Area X, only the former requires NCM. Hence, we conclude that reinforcement learning using WN playback should not be seen as a universally valid paradigm (in adults) for probing neural mechanisms of natural song learning (in juveniles).

Our work contributes to an area of lesion research that allowed, for example, the identification of hippocampal and cortical areas in the initial acquisition of memories (Scoville and Milner, 1957; Teng and Squire, 1999; Squire et al., 2001; Tse et al., 2007) and long-term memory storage (Takehara et al., 2003; Steffenach et al., 2005; Clark et al., 2011), respectively. The ability of NCM-lesioned birds to learn a song discrimination task contrasts with these typically dissociated roles of mammalian brain areas in either acquisition or late retrieval of memories. Reconciling these contrasts could be different neural mechanisms involved in memorization of the target song and of other (nontarget) songs. Also, NCM's memory function may undergo changes from the first critical sensory period to adulthood, reflecting architectural requirements for vocal learning and constraints dictated by critical sensory periods.

While to our knowledge there are no lesion reports in humans that directly parallel our findings, there is an interesting difference between the impairments induced by lesions in the songbird NCM and in the human auditory cortex. Lesions of the latter in the right hemisphere decrease the ability to resolve the direction of pitch changes, but not of pitch differences (Johnsrude et al., 2000). In contrast, NCM-lesioned birds showed a tendency to revert pitch in the direction of baseline, despite not fully restoring baseline pitch. Such tendency could be explained by either the partial nature of our NCM lesions or the partial role of NCM in holding or retrieving the pitch target memory. Our preferred interpretation is the latter: a memory outside NCM might drive pitch in the direction of baseline, whereas the memory in NCM would be needed to precisely match the target. In any case, common to both birds and humans is that different aspects of pitch discrepancy (direction versus distance) seem to be processed in distinct brain regions. While human auditory cortex lesions affect the processing of pitch sensory discrepancy held in shortterm memory, songbird NCM lesions affect the processing of pitch vocal discrepancies in relation to a long-term memory. 


\section{References}

Ali F, Otchy TM, Pehlevan C, Fantana AL, Burak Y, Ölveczky BP (2013) The basal ganglia is necessary for learning spectral, but not temporal, features of birdsong. Neuron 80:494-506. CrossRef Medline

Andalman AS, Fee MS (2009) A basal ganglia-forebrain circuit in the songbird biases motor output to avoid vocal errors. Proc Natl Acad Sci U S A 106:12518-12523. CrossRef Medline

Bolhuis JJ, Gahr M (2006) Neural mechanisms of birdsong memory. Nat Rev Neurosci 7:347-357. CrossRef Medline

Bolhuis JJ, Zijlstra GG, den Boer-Visser AM, Van Der Zee EA (2000) Localized neuronal activation in the zebra finch brain is related to the strength of song learning. Proc Natl Acad Sci U S A 97:2282-2285. CrossRef Medline

Brainard MS, Doupe AJ (2000) Interruption of a basal ganglia-forebrain circuit prevents plasticity of learned vocalizations. Nature 404:762-766. CrossRef Medline

Cherkin A (1972) Retrograde amnesia in the chick: resistance to the reminder effect. Physiol Behav 8:949-955. CrossRef

Chew SJ, Mello C, Nottebohm F, Jarvis E, Vicario DS (1995) Decrements in auditory responses to a repeated conspecific song are long-lasting and require two periods of protein synthesis in the songbird forebrain. Proc Natl Acad Sci U S A 92:3406-3410. CrossRef Medline

Chew SJ, Vicario DS, Nottebohm F (1996) A large-capacity memory system that recognizes the calls and songs of individual birds. Proc Natl Acad Sci U S A 93:1950-1955. CrossRef Medline

Clark RE, Reinagel P, Broadbent NJ, Flister ED, Squire LR (2011) Intact performance on feature-ambiguous discriminations in rats with lesions of the perirhinal cortex. Neuron 70:132-140. CrossRef Medline

Comins JA, Gentner TQ (2010) Working memory for patterned sequences of auditory objects in a songbird. Cognition 117:38-53. CrossRef Medline

Fehér O, Wang H, Saar S, Mitra PP, Tchernichovski O (2009) De novo establishment of wild-type song culture in the zebra finch. Nature 459: 564-568. CrossRef Medline

Fritz J, Mishkin M, Saunders RC (2005) In search of an auditory engram. Proc Natl Acad Sci U S A 102:9359-9364. CrossRef Medline

Funabiki Y, Konishi M (2003) Long memory in song learning by zebra finches. J Neurosci 23:6928-6935. Medline

Gobes SM, Bolhuis JJ (2007) Birdsong memory: a neural dissociation between song recognition and production. Curr Biol 17:789-793. CrossRef Medline

Hahnloser RH, Kotowicz A (2010) Auditory representations and memory in birdsong learning. Curr Opin Neurobiol 20:332-339. CrossRef Medline

Hauser MD, Chomsky N, Fitch WT (2002) The faculty of language: what is it, who has it, and how did it evolve? Science 298:1569-1579. CrossRef Medline

Houde JF, Jordan MI (1998) Sensorimotor adaptation in speech production. Science 279:1213-1216. CrossRef Medline

Immelmann K (1969) Song development in the zebra finch and other estrildid finches. In: Bird vocalizations, pp 61-74. London: Cambridge UP.

Johnsrude IS, Penhune VB, Zatorre RJ (2000) Functional specificity in the right human auditory cortex for perceiving pitch direction. Brain 123: 155-163. CrossRef Medline

Kao MH, Doupe AJ, Brainard MS (2005) Contributions of an avian basal ganglia-forebrain circuit to real-time modulation of song. Nature 433: 638-643. CrossRef Medline

Kojima S, Kao MH, Doupe AJ (2013) Task-related "cortical" bursting depends critically on basal ganglia input and is linked to vocal plasticity. Proc Natl Acad Sci U S A 110:4756-4761. CrossRef Medline

Lane H, Webster JW (1991) Speech deterioration in postlingually deafened adults. J Acoust Soc Am 89:859-866. CrossRef Medline

Leonardo A, Konishi M (1999) Decrystallization of adult birdsong by perturbation of auditory feedback. Nature 399:466-470. CrossRef Medline

Lewis DJ, Misanin JR, Miller RR (1968) Recovery of memory following amnesia. Nature 220:704-705. CrossRef Medline

London SE, Clayton DF (2008) Functional identification of sensory mechanisms required for developmental song learning. Nat Neurosci 11:579586. CrossRef Medline

Marler P, Tamura M (1964) Culturally transmitted patterns of vocal behavior in sparrows. Science 146:1483-1486. CrossRef Medline

Moorman S, Gobes SM, Kuijpers M, Kerkhofs A, Zandbergen MA, Bolhuis JJ
(2012) Human-like brain hemispheric dominance in birdsong learning. Proc Natl Acad Sci U S A 109: 12782-12787. CrossRef Medline

Noll AM (1970) Pitch determination of human speech by the harmonic product spectrum, the harmonic sum spectrum and a maximum likelihood estimate. In: Proceedings of the symposium on computer processing in communications, Vol. XIX, pp 779-797.

Nordeen KW, Nordeen EJ (1992) Auditory feedback is necessary for the maintenance of stereotyped song in adult zebra finches. Behav Neural Biol 57:58-66. CrossRef Medline

Phan ML, Pytte CL, Vicario DS (2006) Early auditory experience generates long-lasting memories that may subserve vocal learning in songbirds. Proc Natl Acad Sci U S A 103:1088-1093. CrossRef Medline

Pinaud R, Terleph TA (2008) A songbird forebrain area potentially involved in auditory discrimination and memory formation. J Biosci 33:145-155. CrossRef Medline

Reiner A, Perkel DJ, Mello CV, Jarvis ED (2004) Songbirds and the revised avian brain nomenclature. Ann N Y Acad Sci 1016:77-108. CrossRef Medline

Romani S, Pinkoviezky I, Rubin A, Tsodyks M (2013) Scaling laws of associative memory retrieval. Neural Comput 25:2523-2544. CrossRef Medline

Scharff C, Nottebohm F (1991) A comparative study of the behavioral deficits following lesions of various parts of the zebra finch song system: implications for vocal learning. J Neurosci 11:2896-2913. Medline

Scott BH, Mishkin M, Yin P (2012) Monkeys have a limited form of shortterm memory in audition. Proc Natl Acad Sci U S A 109:12237-12241. CrossRef Medline

Scoville WB, Milner B (1957) Loss of recent memory after bilateral hippocampal lesions. J Neurol Neurosurg Psychiatry 20:11-21. CrossRef Medline

Squire LR (2006) Lost forever or temporarily misplaced? The long debate about the nature of memory impairment. Learn Mem 13:522-529. CrossRef Medline

Squire LR, Clark RE, Knowlton BJ (2001) Retrograde amnesia. Hippocampus 11:50-55. CrossRef Medline

Standing L (1973) Learning 10,000 pictures. Q J Exp Psychol 25:207-222. CrossRef Medline

Steffenach HA, Witter M, Moser MB, Moser EI (2005) Spatial memory in the rat requires the dorsolateral band of the entorhinal cortex. Neuron 45:301-313. CrossRef Medline

Svirsky MA, Lane H, Perkell JS, Wozniak J (1992) Effects of short-term auditory deprivation on speech production in adult cochlear implant users. J Acoust Soc Am 92:1284-1300. CrossRef Medline

Takehara K, Kawahara S, Kirino Y (2003) Time-dependent reorganization of the brain components underlying memory retention in trace eyeblink conditioning. J Neurosci 23:9897-9905. Medline

Tchernichovski O, Nottebohm F, Ho CE, Pesaran B, Mitra PP (2000) A procedure for an automated measurement of song similarity. Anim Behav 59:1167-1176. CrossRef Medline

Teng E, Squire LR (1999) Memory for places learned long ago is intact after hippocampal damage. Nature 400:675-677. CrossRef Medline

Terpstra NJ, Bolhuis JJ, den Boer-Visser AM (2004) An analysis of the neural representation of birdsong memory. J Neurosci 24:4971-4977. CrossRef Medline

Tokarev K, Tchernichovski O (2014) A novel paradigm for auditory discrimination training with social reinforcement in songbirds. bioRxiv. doi: 10.1101/004176. CrossRef

Tse D, Langston RF, Kakeyama M, Bethus I, Spooner PA, Wood ER, Witter MP, Morris RG (2007) Schemas and memory consolidation. Science 316:76-82. CrossRef Medline

Tumer EC, Brainard MS (2007) Performance variability enables adaptive plasticity of "crystallized" adult birdsong. Nature 450:1240-1244. CrossRef Medline

Warren TL, Tumer EC, Charlesworth JD, Brainard MS (2011) Mechanisms and time course of vocal learning and consolidation in the adult songbird. J Neurophysiol 106:1806-1821. CrossRef Medline

Zann RA (1996) The zebra finch: a synthesis of field and laboratory studies (Oxford ornithology series). New York: Oxford UP.

Zokoll MA, Naue N, Herrmann CS, Langemann U (2008) Auditory memory: a comparison between humans and starlings. Brain Res 1220:33-46. CrossRef Medline 